\title{
Tongue volume and the mandibular dentition
}

\author{
Herman E. Bandy, D.D.S., M.S., and W. Stuart Hunter, D.D.S., Ph.D. \\ Livonia, Mich., and London, Ontario, Canada
}

$\mathrm{O}_{1}$ thodontists long have believed that the tongue and facial musculature play an important role in the establishment of alveolar arch form and in the precise positioning of the teeth. Among the more recent writers taking such a view are Ballard, ${ }^{2}$ Baker, ${ }^{1}$ Graber, ${ }^{5}$ Moss, ${ }^{9}$ and Hovell. ${ }^{7}$ Brodie ${ }^{4}$ is perhaps representative of this group when he writes: "Alveolar bone being as labile as it is, it should be apparent that the teeth would take their positions around the periphery of the tongue and be held in contact with that organ by the forces of the lips and cheeks. One can say with considerable certainty that the dental arch is formed by these two forces."

However, several other workers differ, most notably Brash, ${ }^{3}$ and Scott. ${ }^{31}$ 'Thus, Scott' ${ }^{10}$ pointed out that little support is available for such a concept: "Bone is not so labile as to be completely at the mercy of the adjacent soft tissues. It has its own inherent pattern of growth, and each bone has a shape which varies somewhat from species to species. This shape and its species variation is not merely a result of the balanced effects of the adjacent soft tissues." He further states: "It is, however, more likely that tongue form is determined by the arches enclosing it within the mouth eavity, than that tongue form should determine the normal shape of the arches."

In both cases, the tongue and the dental arches are seen as having a causeand-effect relationship with one another, so that a large tongue might be expected to be associated with large dental arches and vice versa. Since no standardized method of measurement of tongue size or volume exists for the living (Hopkin ${ }^{6}$ has recently described cadaver tongue size), what may be a "large tongue" to one investigator may not be large to another. Therefore, the purpose of this study was to attempt to devise a method of measuring the volume of the tongue

From the Department of Orthodontics and the Center for Human Growth and Development, The University of Michigan.

The major portion of this study was submitted by H.E.B. in partial fulfillment of the requirements for the degree of Master of Science (Orthodontics), The University of Michigan. 
and to explore the relationship of several dental and arch measures with the volume of the tongue in normal subjects.

\section{Method}

In a preliminary pilot study to determine the reliability of subjective estimates of tongue size (whether large, medium, or small) two investigators on two separate occasions could replicate their estimates for less than half of twentyseven test subjects. For only four of these subjects was there agreement both between and within the two investigators on the two separate sets of observations. Attempts to measure the size of the tongue by means of calipers failed because of the mobile nature of the tongue. Alginate impressions of the tongue also proved to be unsatisfactory because of inability to maintain lingual immobility during setting of the material.

A system of fluid displacement was developed, therefore, using a horn-shaped mouthpiece into which the tongue was extended (Fig. 1). The mouthpiece was covered with 0.003 inch rubber-dam material and connected by tubing to an

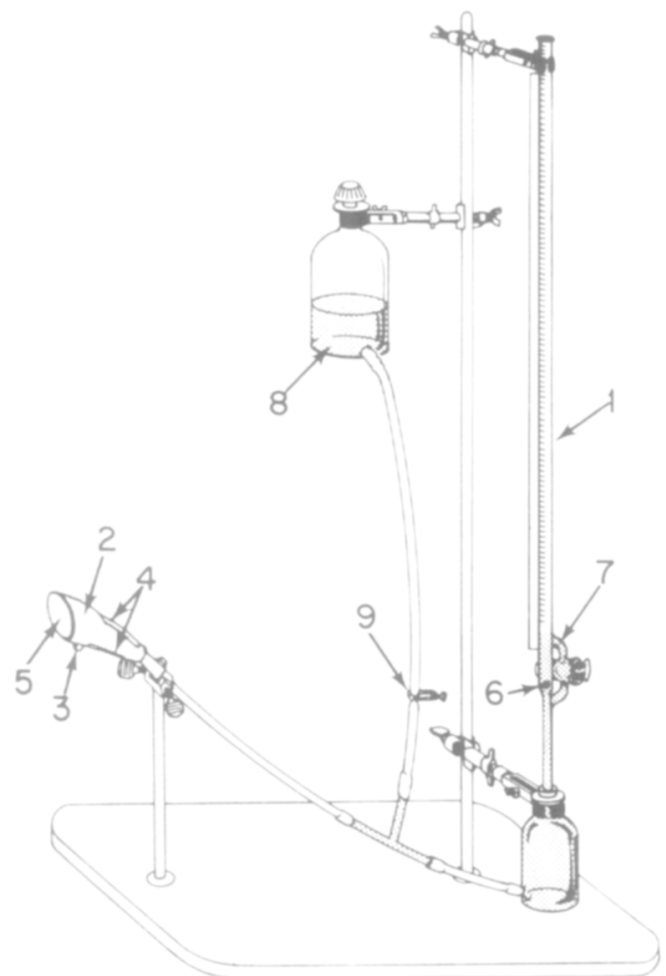

Fig. 1. The apparatus used for measuring tongue volume. 1, Calibrated buret; 2, plastic mouthpiece; 3, stop for mandibular incisors; 4, clear plastic windows; 5, 0.003 inch rubber-dam membrane; 6 , ball valve; 7 , shunt valve; 8 , reservoir; 9, clamp. The clamp for draining the system has been deleted from this drawing for the sake of simplicity. 
open 50 c.c. calibrated buret. A stop for the lower incisors was placed on the mouthpiece $7 \mathrm{~mm}$. ahead of its lowest point. Two transparent windows, one upper and one lower, were inserted in the mouthpiece so that the tongue could be seen during the recording procedure. A radiographic view of the tongue extended into the instrument is shown in Fig. 2 , in which it can be seen that the anterior half of the extended tongue displaces fluid. A plastic millimeter ruler was fused to the lower window so that the amount of extension of the tongue could be read. A ball valve to maintain the leve of the fluid displaced into the buret column during measurement was incorporated, as well as a shunt valve to return the system to zero following each trial. A 500 c.e bottle of water was suspended at the top of the apparatus, serving as a reservoir when refilling the system was required. Four drops of red food coloring were ackled to the water to facilitate reading of the meniscus in the calibrated buret.

To test the aceuracy of volume displacement by this instrument, fire plaster tongues of varying lengths were carred and their displacement was measured in a beaker and in the instrument. The apparatus was found to have an error of between 0.8 and $4.0 \mathrm{per}$ cent, depending on the length of the tongue. The.

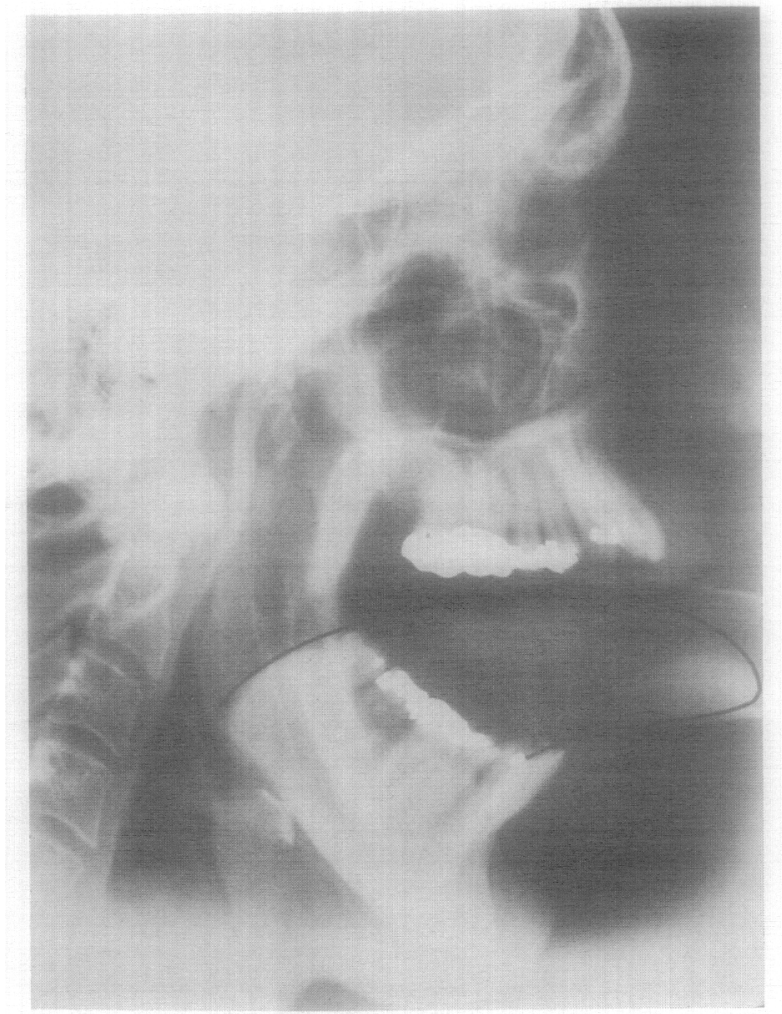

Fig. 2. Cephalometric radiograph of a subject with his tongue protruded into the mouthpiece. The tongue outline has been inked to make it visible in this reproduction. Note the fine wire ligature around the edge of the mouthpiece, running from the upper second molars to the lower incisors. 
longest plaster tongue was larger in diameter at its base than the shortest tongue and more closely fitted the opening of the mouthpiece, so that the membrane adapted itself more closely to the longest plaster tongue, resulting in the smallest percentage of error.

It was found, in measuring human subjects, that the effort involved in protruding the tongue into the instrument must be standardized for each subject. Having the subjects protrude along a millimeter ruler prior to the instrument reading provided an individual achievement goal for subsequent protrusion of the tongue into the instrument.

\section{Testing for accuracy}

To obtain an estimate of the repetitive error of measurement, seventeen subjects werc measured twice, at least one week apart. On each occasion, three volume readings were taken and averaged to give a "reading" for each subject. The mean difference for seventeen subjects between the first and second "readings" was 2.3 c.c., with a standard deviation of 1.4 c.c. Since motivation plays a rather obvious role in obtaining readings, it is doubtful whether significantly more accurate readings could be obtained with this device. In an attempt to improve reliability, however, a series of eight volume readings were made on each subject for the major part of the investigation which follows.

A sample of thirty-nine men, ranging in age from 20 to 37 years, was chosen for our study of the relationship between volume and various arch dimensions.
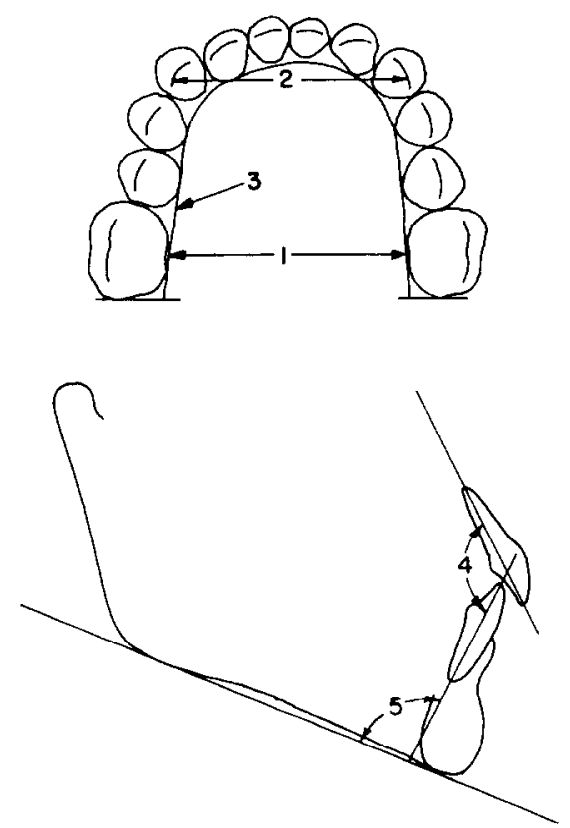

Fig. 3. The measurements made on the mandibular casts at the top and the two measurements taken from the cephalograms at the bottom. 
All subjects hat a full complement of leeth in the lower areh with the exception of the third molars) and no discernible abnomal tongue habits. Tongue length was measured during protrusion on a plastic millinoter ruler. This measurement was made from the labial surface of the most labially placed mandibular incisor to the tip of the tongue. Five such measurements were taken and the arerage was recorled.

A lateral cephalogram was obtained for each subject, and the angles shown in Fig. 3 were measured. The lower aroh dimensions shown in Fig. 3 were obtained from plaster casts. Finally, the subject's tongue rolume was recorded in the newly devised apparatus by having him put his lower ineisors against the stop on the mouthpiece and protrude his tongue into the mouthpiece as far as possible.

After the maximum point to which the tongue could be extended into the mouthpiece was recorded in millimeters, the height of the column of fluid in the calibrated buret was read at the meniseus. Then the amount of extension of the tongue into the mouthpiece was compared with the amount of protrusion on the plastic millimeter ruler previously recorded outside the mouthpiece, to confirm that the subject had extended his tongue as elosely as possible to the original reading. If the difference was greater than $3 \mathrm{~mm}$., the procedure was repeated. However; the instrument length was usually slightly less than actual length because of the resistance of the rubber-dam membrane. The entire procedure was repeated eight times and the values were averaged.

To explore the possibility that scalloping of the lateral borders of the tongue indicates a large tongue, we found seventeen young adult male subjects who showed this condition. Fig. 4 shows the tongue of one such subject.

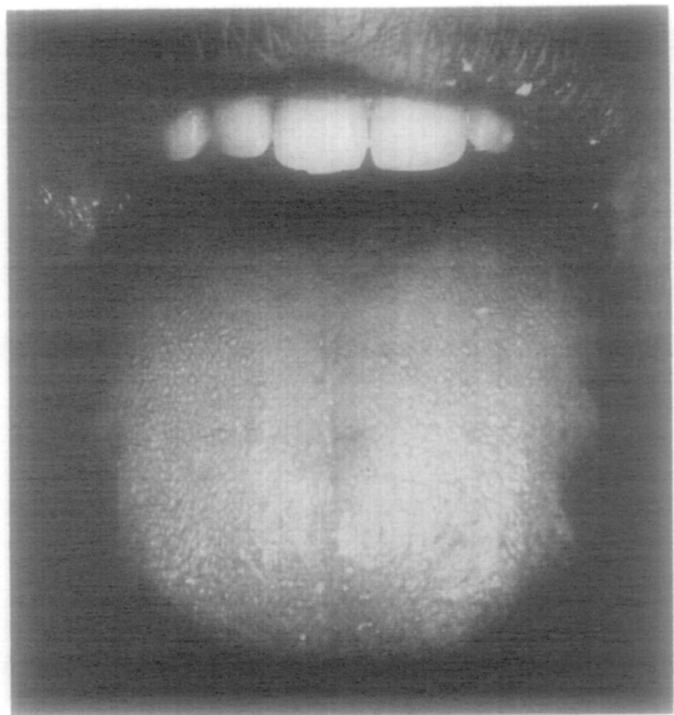

Fig. 4. Photograph of the strongly scalloped tongue of one subject. 


\section{Hypotheses}

The following hypotheses were tested in this study: (1) that this instrument provides a more reliable estimate of tongue volume than a visual appraisal, (2) that tongue length (extensibility) and volume are related, (3) that tongue volume is related to bimolar width, intercanine width, arch perimeter, interincisal angle, and the angle of the incisors to the mandibular plane, (4) that tongue length (extensibility) is related to bimolar width, intereanine width, arch perimeter, interincisal angle, and to the angle of the incisors to the mandibular plane, and (5) that scalloping of the tongue indicates a large tongue.

\section{Findings}

The instrument gave an average error, on repeated readings, of 2.3 c.c. \pm 1.4 c.c., as discussed above. Thus, in the error study of seventeen subjects, in only two cases were the tongues classified differently (small, medium, or large) on the second reading, whereas in the subjective appraisal more than one half of the subjects were appraised differently the second time.

The distribution of tongue volumes as measured by the fluid-displacement method is shown in Fig. 5. The average volume for the sample was 31.4 c.c., with

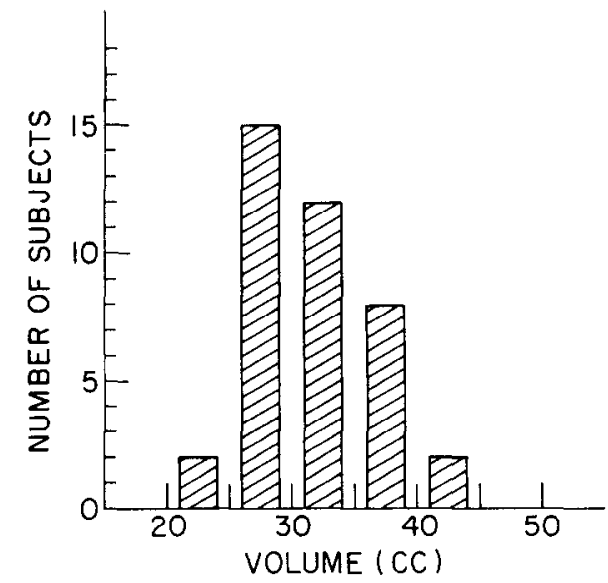

Fig. 5. Bar graph of the distribution of measurable tongue volume for thirty-nine subjects.

Table I. Sample correlation coefficients of measurable tongue volume and other variables $(\mathrm{N}=39$ )

\begin{tabular}{l|c|c}
\hline Tariable Pair & $\begin{array}{c}\text { Correlation } \\
\text { coefficient }\end{array}$ & "t $t$ " test \\
\hline Tongue volume-bimolar width & 0.278 & 1.759 \\
Tongue volume-intercanine width & 0.152 & 0.933 \\
Tongue volume-arch perimeter & 0.376 & $2.465^{*}$ \\
Tongue volume-interincisal angle & -0.178 & -1.088 \\
Tongue volume-angle of incisors to mandibular plane & -0.015 & -0.091 \\
\hline
\end{tabular}

*2.03 or above $=$ Significant at 0.05 level. 
a standard deviation of 4.9 e.c. The extensibility or length of the protruded tongue was found to have a correlation with measurable volume of $0.26(t=1.64)$. which is not statistically significant.

Table I shows the correlation of tongue volume with the other variables measured. It will be noted that only the correlation between measurable tongue volume and arch perimeter is statistically significant at the 0.05 level of confidence.

Table IT shows the correlations of tongue length with the other variables measured. None of these are statistically significant.

Fig. 6 compares the distribution of the tongue volume of scalloped tongues with the main sample of thirty-nine subjects. The average tongue volume for scalloped tongues was 28.6 c.e., with a standard deviation of 3.8 c.e. This is not different statistically from the average of 31.4 c.e. for the main sample.

\section{Discussion}

This method of measurement appears to be more accurate and objective than a visual appraisal of tongue size.

Table II. Sample correlation coefficient of tongue length and other variables $(N=39)$

\begin{tabular}{l|r|r}
\hline \multicolumn{1}{c|}{ Variable Pair } & $\begin{array}{c}\text { Correlation } \\
\text { cocfficient }\end{array}$ & " $t$ " test \\
\hline Tongue length (actual)-bimolar width & -0.110 & -0.673 \\
Tongue length (actual) -intercanine widtl & 0.115 & 0.705 \\
Tongue length (actual) -arch perimeter & -0.106 & -0.646 \\
Tongue length (actual) -interincisal angle & 0.146 & 0.887 \\
Tongue length (actual)-angle of ineisors to mandibular plane & -0.209 & -1.279 \\
\hline
\end{tabular}

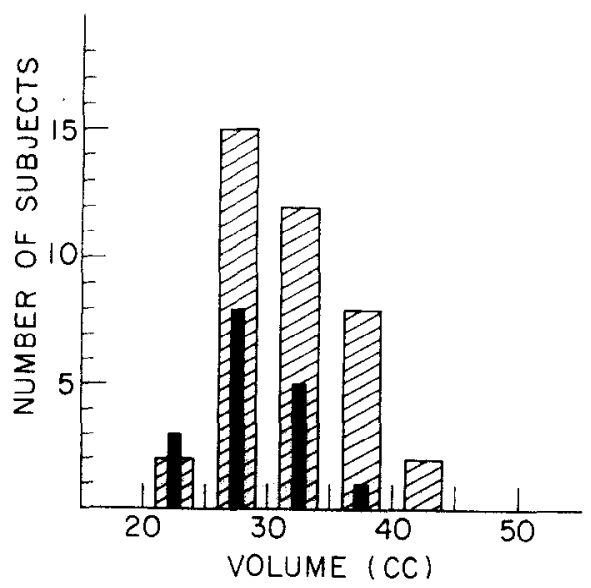

Fig. 6. Bar graph of the measurable volume for the tongues of seventeen subjects with scalloping (in black) superimposed on the bar graph of the subjects in the main sample. (See Fig. 5.) 
As stated in the second hypothesis, one might expect a longer tongue to have greater volume than a shorter tongue. However, according to the findings of this study, such is the case only to an insignificant extent, at. least in normal subjects. This method of measuring tongue volume accounts for three dimensions of the extended tongue, of which extensibility or measurable length is only one. Width and thickness factors appear to be equally important.

With regard to the third hypothesis (Table $I$ ), four of the variables related to measurable tongue volume (bimolar width, intercanine width, interincisal angle, and arch perimeter) increase or decrease with tongue volume. However, the size of the correlation coefficients suggests caution in drawing inferences from these relationships. Only one correlation, that with arch perimeter, is statistically significant at the 0.05 level. Such a relationship is of limited clinical utility. However, it is of interest to note that arch perimetcr combines two dimensions, thus approximating the three dimensions of the tongue to a greater extent than the other measures. The size of the correlation of incisor angle to mandibular plane with measurable tongue volume, for all practical purposes, may be considered zero.

With regard to the fourth hypothesis (Table II), of the five variables tested with protruded tongue length, only one (intercanine width) increases with increasing tongue length. It might be expected that an increase in protruded length of the tongue would coincide with a decrease in the interincisal angle and an increase in the arch perimeter and the angle of the lower incisors to the mandibular plane. This was not the case, and the relationship in all five measures is sufficiently low as to be essentially random. Again, protruded tongue length is but one of three pertinent variables. Width and thickness are also important.

The sample of subjects with scalloped tongues showed no difference from the main sample, thus refuting the hypothesis that scalloping indicates a large tongue.

The low correlation coefficients found between the paired variables thus suggest that, at least within a normal range of tongue volumes, a large tongue can be found with either large or small dental arches and that a small tongue may also be found with either large or small dental arches. The findings may also be seen as supporting Brash, ${ }^{3}$ who said: ". . . it is improbable that the tongue exercises any important direct mechanical influence on the general form and size of the mandible. . . ." They also tend to support Scott," who said: "The primary form of the alveolar processes and dental arches is determined before birth prior to the eruption of the teeth and independent of muscle activity. ... The postnatal development of the alveolar processes and dental arches is such that arch form is maintained in spite of the greater pressure exerted by the tongue as compared with the lips and cheeks."

Thus, there appears to be no close relationship between tongue volume and lower arch dimensions, incisor angulation, tongue length, or scalloping of the tongue, at least in the sample of normal adult males studied here.

This was a study simply of volume. However, it has been assumed that the density of the tongue does not vary markedly from person to person; nor have lingual pressures been measured. Recent investigations by Lear ${ }^{8}$ show that tongue 
and cheek pressures seldom balance precisely. Thus, neither the volume nor tho pressure of the tongue appears to have as great an influence on the size of the dental arch as was thought.

\section{Summary}

A method utilizing the prineiple of fluid displacement was devised to measure the volume of the anterior portion of the tongue.

A sample of thirty-nine men was used to test the relationships betwern measurable tongue volume, tongue length, certain angular cephalometric measurements, and lower arch dimensions. Seventeen subjects showing scalloping of the lateral borders of the tongue were compared with the main sample.

Conclusions from this investigation are as follows:

1. The volume of the anterior portion of the tongue can be measured with an average accuracy of 2.3 c.e. \pm 1.4 c.c., using the instrument described here.

2. In this sample, and with this method of measurement, the volume and length of the tongue seem to have little, if any, influence on the width and length of the lower dental arch, on the degree of interincisal relationship, and on the angle of the lower incisor teeth to the mandibular plane.

3. A statistically significant correlation of 0.4 exists between measurable tongue volume and arch perimeter.

4. Sealloping of the tongue does not appear to be an indication of a large tongue.

The authors are grateful to Professor Robert F. Moyers for his encouragement in this sturly and to the Class of 1968 (Dentistry) for their willingness to participate in the study.

\section{REFERENCES}

1. Baker, R. E.: The tongue and dental function, AM. J. ORTHodontics 40: 927-924, 1954.

2. Ballard, C. F.: The significance of soft tissue morphology in diagnosis and treatment planning, Tr. European Orthodont. Soc., pp. 143-169, 1953.

3. Brash, J. C., Mckeag, H. T. A., and Scott, J. H.: The aetiology of irregularity and maloeclusion of the teeth, London, 1956, Dental Board of the United Kingdom, p. 240.

4. Brodie, A. G.: Thoughts on the aetiology of malocelusion, Tr. European Orthodont. Soe. 33: $200 \cdot 215,1957$.

5. Graber, T. M.: Orthodonties, Principles and Practice, ed. 2, Philadelphia, 1966, W. B. Naunders Company, p. 317.

6. Hopkin, G. B.: Neonatal and adult tongue dimensions, Angle Orthodontist 37: 132-133. 1967 .

7. Hovell, J. H.: The relationship of the oro-facial musculature to occlusion: Current British thought. In Kraus, B. S., and Riedel, R. A. (editors): Vistas in orthodonties, Philadelphia, 1962, Lea \& Febiger, pp. 328-345.

s. Lear, C. S. C.: Symmetry analyses of the palate and maxillary dental arch, Angle? Orthodontist 38: $56-62,1968$.

9. Moss, M. L.: The functional matrix. In Kraus, B. S., and Riedel, R. A. (editors) : Vistas in orthodontics, Philadelphia, 1962, Lea \& Febiger, pp. 85-98.

10. Scott, T. H.: The role of soft tissue in determining normal and abnormal dental occlu. sion, D. Practitioner \& D. Record 11: 302-308, 1961. 